\title{
MODELS AND AN ALGORITHM FOR MULTI-CRITERIA SYNTHESIS OF CONTROL TECHNOLOGIES MANAGING INFORMATION SYSTEMS OF VIRTUAL ENTERPRISES
}

\author{
Dmitry A. Ivanov \\ Chemnitz University of Technology \\ Department of Economics and Business Administration \\ Chair of Production and Industrial Management \\ D-09107 Chemnitz, Germany \\ E-Mail: idm@hrz.tu-chemnitz.de \\ Boris V. Sokolov, Dmitry N. Verzilin, Evgeniy M. Zaychik, \\ Russian Academy of Science, \\ Saint Petersburg Institute of Informatics and Automation \\ 39, 14 Linia, VO \\ St.Petersburg, 199178, Russia \\ E-mail: sokol@iias.spb.su,verzilin@SV10100.spb.edu,EZaychik@beeline.ru,
}

\section{KEYWORDS}

Optimization, Planning, Dynamic Models, Simulation, Information Systems, Virtual Enterprises.

\begin{abstract}
Integrated information systems (IIS) of virtual enterprises (VE) were considered as objects of control. Two interrelated problems were jointly stated. The first problem lied in program management of IIS and ranking its structural states. The second one implied that control functions regulating business processes should be reallocated among elements and subsystems of IIS in a real time mode. A formal multi-criteria description of these problems was made and a combined (simulationbased) algorithm of a solution was worked out.
\end{abstract}

\section{INTRODUCTION}

Virtual enterprises unite independent multi-business partners (real enterprises) within a temporal taskoriented technical-organizational structure through information technologies and telecommunications (Camarihna-Matos et al. 2004, Wang and Norrie 2001, Ivanov 2003). Virtual enterprises are highly adaptive to consumer needs and benefit juridical and physical persons providing them with dynamic use of common resources during remote collaboration within a business project.

A virtual enterprise is a typical example of a modern integrated transport, production and trading network performing intensive structural dynamics. This issue makes the structural synthesis of a VE more complicated. In particular, the structure dynamics have a complicative influence upon the following tasks: partner selection (producers and suppliers of components, retailers, etc); end products configuring; placement of orders; configuring transport network and information resources (Camarihna-Matos et al. 2004, Wang and Norrie 2001).

An integrated information system is one of the main subsystems of VE. It should be constructed through a real-time configuring (structure-functional synthesis) and interconnection of individual information systems belonging to participants (real enterprises).

It is obvious that IIS run under conditions of structure dynamics same as VE does (Okhtilev et al 2006). Possible variants of structure dynamics involving modern information systems are illustrated in fig. 1. Our previous investigations confirmed that incrementation (stabilization) of IIS potentialities and capacity for work necessitates structures control (including the control of IIS structures reconfiguration). There are many possible variants of structure-dynamics control suitable for IIS. The following variants are the most typical: alteration of IIS functioning means and objectives; controlled motion of CTS elements and subsystems; alteration of the order of IIS tasks; redistribution of functions, tasks, control algorithms and information flows among IIS levels; reconfiguring of degraded structures; flexible use of reduced technologies of IIS control.

As applied to IIS, the structure-dynamics control belongs to the general discipline of structure-functional synthesis and program construction, provided for IIS development (Okhtilev et al 2006, Kalinin and Sokolov 1995, Zvirkun and Akinfiev 1993, Zvirkun et al. 1985, Zimin and Ivanilov 1971).

Here we consider an important problem of structuredynamics control including interrelated business processes (BP) planning and planning of IIS operations aimed at BP improvements. 


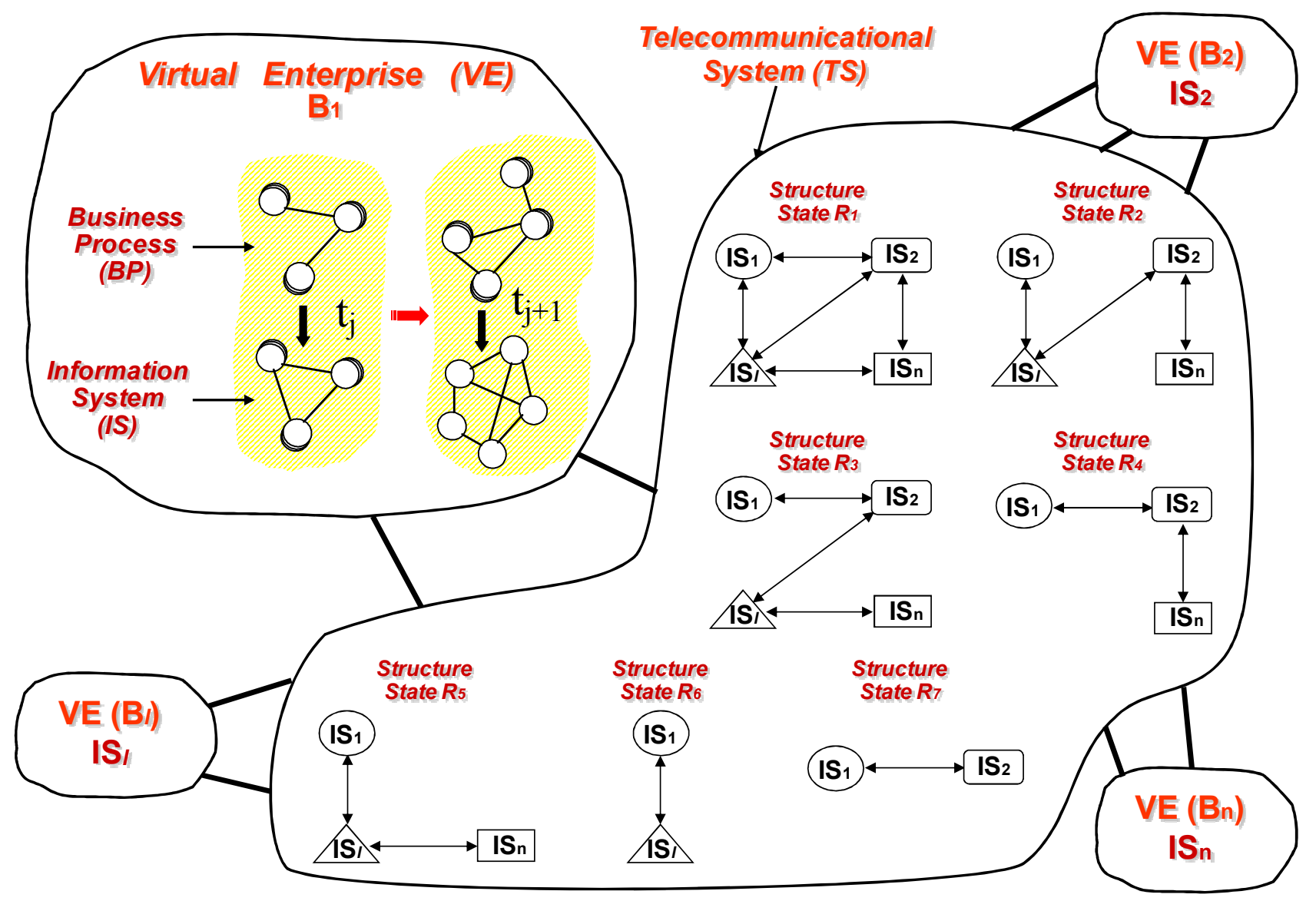

Figures 1: Variants of structure dynamics

\section{PROBLEM STATEMENT}

Modern IIS ought to reconfigure and adjust information processes in order to agree with changeable business projects and conditions of their execution.

Efficient functioning of IIS necessitates flexible redistribution of tasks, functions and algorithms among elements, subsystems and levels of the system. Therefore following main tasks have to be redistributed: receiving, transmission and processing of information, planning and control of IIS and VE operation. Moreover, different control technologies involve different variants of IIS structures and different information flows in control loops of IIS.

From a formal point of view a selection (synthesis) of BP structure as well as a real-time structure-functional synthesis of IIS implies joint multi-criteria optimization of VE and IIS operation, selection of control functions for business processes and redistribution of control functions among nodes of IIS. Due to the real-time mode of the IIS, the problem to be solved is more complicated than the ones described in the works of (Zvirkun and Akinfiev 1993, Zvirkun et al. 1985).

The problem of real-time distribution of control functions can be solved at different stages of BP life cycle. In our approach we consider the period of IIS operation planning with simultaneous preliminary distribution of control functions among main elements of IIS and with construction of control programs for these elements. The control programs can be corrected at the stage of real-time control (implementation of the plan). Program corrections can be accompanied by reallocation of resources and by reconfiguration of IIS structures.

Let us introduce some notation for problem definition. Let $A=\left\{A_{i}, i \in N=\{1, \ldots, n\}\right\}$ be a set of business processes (and corresponding control functions) to be implemented at some node of IIS at a given time interval $T=\left[t_{0}, t_{f}\right]$. To achieve the VE goals during the interval $\mathrm{T}$, the BPs have to be fulfilled. We distinguish between the functions of goal definition, planning (long term and operational planning), real-time control, VE states analysis, external situation analysis and coordination. The set $A=\left\{A_{i}, i \in N\right\}$ is related to sets of informationaltechnological operations $D^{(i)}=\left\{D_{æ}^{(i)}, \mathfrak{x} \in K=\left\{1, \ldots, s_{i}\right\}\right\}, \quad$ that are necessary for implementation of BP $A_{i}, i=1, \ldots, n$. Let $B=\left\{B_{j}, j \in M=\{1, \ldots, m\}\right\}$ be a set VE main elements and subsystems. Each element $B_{\mathrm{j}}$ can include technical facilities $\quad C^{(j)}=\left\{C_{\lambda}^{(j)}, \lambda \in L=\{1, \ldots, l\}\right\} \quad$ with 
appropriate computer equipment and software. Technical facilities are used for implementation of control functions.

Let $E(t)=\left\|e_{i}(t)\right\|$ be a known matrix function, with $e_{i j}(t)=1$ in case of the subsystem $B_{j}$ is carrying out the function $A_{i}$ at time $t$ in accordance with time-spatial, technical and technological constraints, $e_{i j}(t)=0$ otherwise.

Fig.1 presents an example of seven possible structural states of IIS. The arrows show variants of communication within the system. These variants correspond to different control technologies (methods of IIS application) and different spatial structures of VE.

Now the verbal description of a functions-distribution problem can be presented as follows. It is necessary to select the best variants of functions distribution among the nodes of IIS for each structural state $R_{l}, R_{2}, \ldots, R_{k}$ of IIS (under known time spatial, technical and technological constraints) and to find the best variants of functions implementation. The structural states of IIS should be sorted according to their preference. The preference relation can be expressed through quality functions characterizing efficiency of IIS and its structural and technologic characteristics.

The described problem belongs to the class of multicriteria choice problems with finite sets of alternatives (structural states of IIS).

\section{ALGORITHM OF MULTI-CRITERIA PLANNING OPERATIONS IN IIS}

The general algorithm for the problem includes the following steps.

Step 1. Models (analytical, simulation and combined models) describing structural states $R_{1}, R_{2}, \ldots, R_{k}$ are used for optimal distribution of $\mathrm{BP}$ and control functions among subsystems of IIS, for technological operations planning and for evaluation of IIS efficiency. The following characteristics of IIS efficiency can be used: the total number of functions implemented in subsystems during the interval $\mathrm{T}$, the total number of $\mathrm{BP}$ in given macro-states, the total number of technological operations executed over the time interval $\mathrm{T}$, the total time of operations over the time period T. The abovementioned characteristics can have stochastic or fuzzy interpretation if uncertainty factors are present (Okhtilev et al 2006, Orlovski. 1981).

The following dynamic model of functions distribution can be used for evaluation of IIS efficiency (Okhtilev et al 2006, Kalinin and Sokolov 1995, Zimin and Ivanilov 1971).

$$
\begin{aligned}
& \dot{x}_{i}^{(\phi)}=\sum_{j=1}^{m} \varepsilon_{i j}(t) u_{i j}^{(\phi)} ; \dot{x}_{i \mathfrak{x} j}^{(0)}=\sum_{\lambda=1}^{l} b_{i æ j \lambda} u_{i æ j \lambda}^{(0)} ; \\
& \dot{y}_{i j}^{(\phi)}=v_{i j}^{(\phi)} ; \\
& \sum_{j=1}^{m} u_{i j}^{(\phi)}\left[\sum_{\alpha \in \Gamma_{i 1}}\left(a_{\alpha}^{(\phi)}-x_{\alpha}^{(\phi)}\right)+\prod_{\beta \in \Gamma_{i 2}}\left(a_{\gamma}^{(\phi)}-x_{\gamma}^{(\phi)}\right)\right]=0 ;
\end{aligned}
$$

$\sum_{\lambda=1}^{l} u_{i æ j \lambda}^{(0)}\left[\sum_{v \in \Gamma_{i æ 1}}\left(a_{i v j}^{(0)}-x_{i v j}^{(0)}\right)+\prod_{\mu \in \Gamma_{i æ 2}}\left(a_{i \mu j}^{(0)}-x_{i \mu j}^{(0)}\right)\right]=0 ;(3)$

$\sum_{i=1}^{n} u_{i j}^{(\phi)}(t) \leq 1 ; \forall j ; \sum_{j=1}^{m} u_{i j}^{(\phi)}(t) \leq 1 ; \forall i$

$u_{i j}^{(\phi)}(t) \in\{0,1\}$

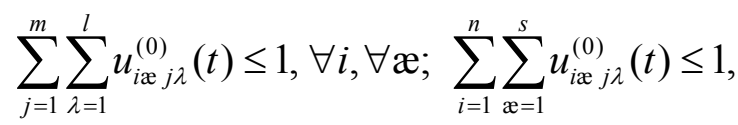

$\forall i, \forall æ ; u_{i æ j \lambda}^{(0)}(t) \in\left\{0, u_{i j}^{(\phi)}\right\} ;$

$v_{i j}^{(\phi)}\left(a_{i s j}^{(0)}-x_{i s j}^{(0)}\right)=0 ; v_{i j}^{(\phi)}(t) \in\{0,1\}$

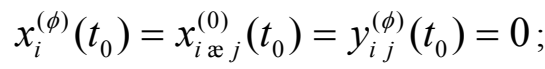

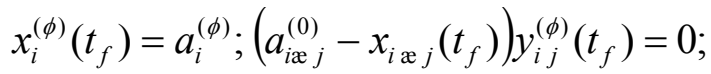

$x_{i}^{(\phi)}\left(t_{f}\right)=a_{i}^{(\phi)} ;\left(a_{i æ j}^{(0)}-x_{i æ j}\left(t_{f}\right)\right) y_{i j}^{(\phi)}\left(t_{f}\right)=0 ;$

$J_{0}=\sum_{i=1}^{n} \sum_{j=1}^{m} v_{i j}^{(\phi)}\left(t_{f}\right) ; J_{1}^{(n)}=\sum_{i=1}^{m} v_{n j}^{(\phi)}\left(t_{f}\right) ;$

$J_{0}=\sum_{i=1}^{n} \sum_{j=1}^{m} v_{i j}^{(\phi)}\left(t_{f}\right) ; J_{1}^{(n)}=\sum_{i=1}^{m} v_{n j}^{(\phi)}\left(t_{f}\right) ;$

where $x_{i}^{(\phi)}(t)$ is equal to total duration of the business process $A i$ fulfillment in subsystem $B j$ as $u_{i j}^{(\phi)}(t)=1$;

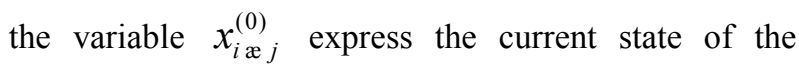
technological operation $D_{\mathfrak{x}}^{(i)} ; y_{i j}^{(\phi)}$ is equal to the time passed after $A_{i}$ completion in $B_{j}$ until the time $t=t_{f}$; $a_{\alpha}^{(\phi)}, a_{\alpha}^{(0)}, a_{\gamma}^{(0)}, a_{i v j}^{(0)}, a_{i \mu j}^{(0)}$ are given values setting end conditions

for $x_{i}^{(\phi)}(t), x_{\alpha}^{(\phi)}(t), x_{\gamma}^{(\phi)}(t), x_{i v j}^{(0)}(t), x_{i \mu j}^{(0)}(t)$ at time $t=$

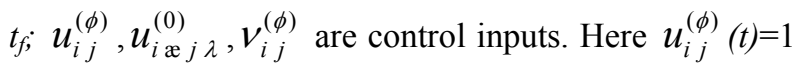
if BP $A_{i}$ is being executed in the subsystem $B_{j}$ at time $t$, $u_{i j}^{(\phi)}(t)=0$ otherwise; $u_{i æ j \lambda}^{(0)}(t)=1$ if the technological operation $D_{\mathfrak{x}}^{(i)}$ is executed in the technical facility $C_{\lambda}^{(j)}, u_{i æ j \lambda}^{(0)}(t)=0$ otherwise; $v_{i j}^{(\phi)}=1$ if $\mathrm{BP} A_{i}$ was implemented in the subsystem $B_{j}, v_{i j}^{(\phi)}=0$ otherwise. Here the sets $\Gamma_{i 1}, \Gamma_{i 2}$ include the numbers of functions that are direct predecessors of the control function Ai. The set $\Gamma_{i 1}$ indicates predecessors connected by logical "and", the set $\Gamma_{i 2}$ indicates 
predecessors connected by logical “or". The sets $\Gamma_{i \text { æ1 }}$, $\Gamma_{i \text { æ2 }}$ include the numbers of technological operations $D_{v}^{(i)}$ and $D_{\mu}^{(i)}$ that are direct predecessors of the operation $D_{\mathfrak{x}}^{(i)}$. The subscripts 1 and 2 express the type of logical connection as stated above.

Therefore, constraints (2) and (3) define allowable sequences of control functions and technological operations. Constraints (4) and (5) specify that each BP at each time can be carried out only in one subsystem $B_{j}$ $(i=1, \ldots, n ; j=1, \ldots, m)$ and conversely, each subsystem $B_{j}$ can carry out only one BP $A_{\mathrm{i}}$ at the same time. Similar constraints are used for technological operations $D_{\mathfrak{x}}^{(i)}$ that are executed at the technical facility $C_{\lambda}^{(j)}$.

Expression (6) states switching-on conditions for the auxiliary control input $v_{i j}^{(\phi)}(t)$. Expressions (7) and (8) specify end conditions for the state variables at the time $t=t_{0}, t=t_{f}, \mathrm{R}^{1}$ is a set of positive real numbers. The functionals $J_{0}, J_{1}, J_{2}$ are quality measures for distribution of $\mathrm{BP}$ in IIS. Here $J_{0}$ is equal to total number of functions by the time $t=t_{f}, J_{l}$ is equal to the number of subsystems the function $A_{i}$ is implemented in, $J_{2}$ expresses the elapsed time for implementation of all necessary functions.

A simulation model of real-time control can be used together with expressions (1)-(9) for taking into account uncertainty factors. In this case special procedures of inter-model coordination can be used (Okhtilev et al 2006, Kalinin and Sokolov 1995).

Extreme values of functionals characterizing IIS efficiency can be determined via solution of optimal control problem for finite-dimensional differential system with mixed conditions. The solution algorithms and different aspects of their programming are considered in (Okhtilev et al 2006, Kalinin and Sokolov 1995).

Step 2. Structure-topological characteristics of IIS are being evaluated (Zimin and Ivanilov 1971) including: the coefficient of attainability $J_{4}$, different measures of structure compactness (radius $J_{5}$ of the structure, diameter $J_{6}$ of the structure, integral measure $J_{7}$ of structural compactness), measures $J_{8}$ of structure centralization (decentralization).

The formulas for computation of measures are proposed in (Zimin and Ivanilov 1971).

Step 3. The pairwise-comparison matrix $K c$ is completed for measuring the IIS efficiency. Expert appraisal is used for completion of the matrix.

Step 4. The weights of measures (significance coefficients) are evaluated according to the matrix $K c$. The algorithm proposed in (Orlovski 1981) is used here. The vector of coefficients is equal to the normalized eigenvector $\vec{\omega}_{\mathrm{c}}$ corresponding to the maximal eigenvalue Lmax of the matrix $K c$. Thus the following equation has to be solved:

$$
\left(K c-\operatorname{Lmax}^{*} I\right) \vec{\omega}_{\mathrm{c}}=0,
$$

where $I$ is a unitary matrix.

Then a weight of each structural state $\left(R_{l}, R_{2}, \ldots, R_{k}\right)$ of IIS for each measure taken separately is evaluated. These weights complete the matrix $K r$. Each column of the matrix $K r$ includes relative weights of the states in respect of some measure. A weighted sum of measures is received for each alternative $R_{1}, R_{2}, \ldots, R_{k}$. In other words, total sets of weights are determined for each structural state via the formula:

$$
\operatorname{Kr} \vec{\omega}_{\mathrm{c}}=\vec{\omega}^{*}
$$

Step 5. The structural states are sorted according to their preference. The best one is characterized with the maximal element of the vector $\vec{\omega}^{*}$. Each element of the vector $\vec{\omega}^{*}$ can be interpreted as a total weight of some structural state.

\section{CONCLUSIONS}

Dynamic interpretation of operation planning in IIS let thoroughly describe and investigate interrelation and interaction of business processes and the processes of information processing, storing and interchange.

The framework of integrated multi-criteria operations planning in the context of IIS structure-dynamics control results in the following advantages. The goals of IIS planning can be directly interrelated with the goals of business processes. Structure-dynamics operations (IIS control technology) can be reasonably selected and substantiated. Efficient compromise solutions can be found for allocation of control functions among the elements of IIS and for general programs (plans) of IIS operation. The preliminary ordering of IIS structural states let rapidly reconfigure it in case of failures (Okhtilev et al 2006).

Several prototype versions of software were produced for structure-dynamics control of IIS in different application domains (cosmonautics, power industry, management, etc, see http://www.spiiras-grom.ru). The experiments with software confirmed efficiency of models applied.

This work was supported by Russian Foundation for Fundamental Investigations (grants 06-07-89242, 07-0700169, 08-08-00403), by Department of Information Technologies and Computing of Russian Academy of Sci. (Project 2.5).

\section{REFERENCES}

Camarihna-Matos, L., Kluwer (editors) et al 2004. Virtual Enterprises and Collaborative Networks, Academic Publishers, $610 \mathrm{p}$.

Wang L. and Norrie D. H. 2001. "Process Planning and Control in a Holonic Manufacturing Environment." Journal of Applied Systems Studies, 2(1), 106-126.

Ivanov, D.A. 2003 Virtual Enterprises and Logistics Chains: Integrated Approach to Organization and Control in New Forms of Production Cooperation SPbSUEF, 120 p. (In Russian) 
Ohtilev, M.Yu., Sokolov, B.V., Yusupov, R.M. 2006. Intellectual Technologies for Monitoring and Control of Structure-Dynamics of Complex Technical Objects. Moscow, Nauka, 410 p. (in Russian)

Kalinin, V.N. and Sokolov, B.V. 1995. "Multiple-Model Approach to Description of Control Processes in Space Systems." Control Theory and Systems, No 1, 149-156. (In Russian).

Zvirkun, A.D. and Akinfiev, V.K. 1993. Structure of the Multi-Level Systems (Synthesis and Development). Moscow, Nauka. (in Russian).

Zvirkun, A.D. and Akinfiev, V.K., Filippov, V.A. 1985. Simulation Modeling in the Problems of Complex Systems Structure Synthesis. Moscow, Nauka, 172 p. (in Russian).

Zimin, I.N. and Ivanilov Yu.,P. 1971. "Solving of Network Planning Problems via a Reduction to Optimal Control Problems." Journal of Calculus Mathematics and Mathematical Physics. 11, No 3, 632-641 (in Russian).

Ore, O. Theory of Graphs. 1962. AMS Colloquium Publications Vol. 38, AMS, Providence, RI, 270 p.

Orlovski, S.A. 1981. Decision Making under Fuzzy Information. Moscow, Nauka, 208 p. (in Russian).

\section{AUTHOR BIOGRAPHIES}

DMITRY IVANOV is a researcher at the Chemnitz University of Technology and Chair of the GermanRussian Coordination Office for Logistic. He studied production management and engineering (2000). In 2002, he graduated in Saint Petersburg as a Ph.D. in Economics on the topic of operative supply chain planning and control in virtual enterprises. In 2006, he received the Dr.rer.pol. degree at the Chemnitz University of Technology. He is an author of six scientific books and more than 70 papers published in international and national journals, books and conference proceedings. Since 2001, he has been involved in research and industry projects on supply chain management and virtual enterprises. Dr. Ivanov received a German Chancellor Scholarship Award in 2005. His e-mail address is : idmehrz.tu-chemnitz.de.

BORIS V. SOKOLOV was born in Leningrad (now Saint-Petersburg), Russia in 1951. He obtained his main degrees in Mozhaisky Space Engineering Academy, Leningrad. MS in Automation Control Systems of Space Vehicles in 1974. Candidate of Technical Sciences subject the area of planning automation and decision making in 1982. Doctor of Technical Sciences subject the area of military cybernetics, mathematical modeling and methods in military research. Professional Interests: Basic and applied research in mathematical modeling and mathematical methods in scientific research, optimal control theory, mathematical models and methods of support and decision making in complex organizationtechnical systems under uncertainties and multi- criteria. At present he is a deputy director of St.-Petersburg Institute for Informatics and Automation. His e-mail address is: sokoleiias.spb.su and his Web-page can be found at http: //www.spiiras-grom.ru.

DMITRY N. VERZILIN was born in Leningrad (now Saint-Petersburg), Russia in 1960. He graduated from
Mathematical faculty of Leningrad States University in 1982. He obtained the degree of Candidate of Technical Sciences in Mozhaisky Space Engineering Academy, 1992 and the degree of Doctor of Economics in St.Petersburg States University of Economics and Finances, 2004. At present he is a leading researcher of St.-Petersburg Institute for Informatics and Automation. Professional interests lay in operations research, simulation, and statistical analysis. His e-mail address is verzilinesv10100.spb.edu.

EUGENIY M. ZAYCHIK was born in 1962 in Rostovon-Don, Russia. He graduated from Military Academy of Communication in 1992. He is a Candidate of Technical Sciences (1994, Military Academy of Communication). He is a specialist in simulation and control of communication systems. At present he works in St.-Petersburg Institute for Informatics and Automation. His e-mail address is EZaychik@beeline.ru. 